\title{
HeALTh EdUCATION NEEDS AMONG INDIVIDUALS WITH LOW BACK PAIN
}

\begin{abstract}
Lack of positive results from many of the health-education programmes for patients with low-back pain (LBP) is possibly due to the type of health information that may have been presented and the method that had been used. The present study sought to explore health-education needs among individuals with LBP. A qualitative approach that utilised in-depth interviews and a focus-group discussion was used. Data was drawn from ten participants attending physiotherapy treatment due to a non-specific LBP at the Nairobi Hospital Rehabilitation Unit, using purposive sampling. A thematic analysis procedure was used to analyse the data. The study found the participants' health education needs to be incongruent with the medical professionals' assumptions of what the patients' health education needs were. Deficiencies in explanation of the cause, diagnosis, prognosis, and the appropriate use of health services were found. The findings suggest that a more encompassing model of health education was believed to be more fitting. Since the findings indicate that the individuals with LBP require health education on various aspects, a Rehabilitative Model of health education was probably more pertinent to the individuals. That way, a more encompassing, all-inclusive model of health education would cover on the aspects that were currently neglected.
\end{abstract}

KEY WORDS: BACK PAIN, HEALTH EDUCATION, PATIENTS, NEEDS.

\section{INTRODUCTION}

Due to the multifactorial nature of lowback pain, efforts aimed at preventing and managing the prevalence of the condition has proven mostly unsuccessful. Effective treatment of low-back pain is indeed embraced by substantial difficulties (Linton and van Tulder 2001, Harland and Lavallee 2003). This has led to the existence of many therapeutic and health-education approaches (Moseley 2002). As a result, several guidelines (protocols) have been developed to provide a template for more effective clinical practice on low back pain management (Bigos et al 1994, Li and Bombardier 2001). Although the guidelines recommend patient education as a means of limiting some of the problems experienced by the patients suffering from low back pain (Bigos et al 1994), none has been found to be a model for these patients.

CORRESPONDENCE TO:

Nyagah Julius Ng'uurah,

Rehabilitation Unit,

Nairobi Hospital

P.O Box 30026-00100

Nairobi Kenya

Email: jnnyagah@yahoo.com.

Tel: +254 (722) 700949

$+254(020) 2714400$
Furthermore, for some time now, there has been a consensus of opinion that there was no clinically-significant achievement of health-education programmes for low-back pain (Cohen et al 1994). Additionally, reviews of education-based 'back' schools provide convincing evidence that patient education has failed to effect any positive changes on low-back pain sufferers (Koes et al 1994). However, the latest evidence implies that the lack of positive effects in many of the health-education programmes was probably due to the type of education that had been presented to patients and the methods used to present the information (Mosley 2002). The health education that has mostly been presented to patients has always been planned, designed, and implemented by the medical professionals without their taking into consideration the views and opinions from the consumers of this information (Axelsson et al 1995). For example, written patient education has always been based on the medical professionals' assumption of what patients needed to know. These assumptions have frequently been shown to be incompatible with the patients' actual needs (Axelsson et al 1995, Coulter et al 1998).

Since physiotherapists work principally within a biomedical paradigm, health is seen as an absence of disease, while affective or cognitive parameters of health are usually ignored (Richardson and Eastlake 1994). This assumption obviously neglects other aspects that evidence has proved as influencing the development and sustenance of LBP. Psychosocial, economic, environmental, and occupational aspects among others do play a role in influencing the development and sustenance of LBP. When these aspects are ignored during health education sessions, it could possibly result in LBP progressing into the chronic stage. In addition, the Medical Model of health education had failed to take into consideration the individuals' needs that were likely to influence negatively LBP during the management of the problem. This approach views LBP as a predicament of the individual, clearly caused by a disease, trauma, or other health situations requiring therapeutic care provided only in the form of the patient's treatment medically, surgically, or physically by the professionals (WHO 2001). Published research on low-back pain has similarly utilised the biomedical model of tissue dysfunction that focuses on disease and tissue pathology rather than on illness (Bardin 2002) or on the problems individuals experience as they manage 
low-back pain. However, according to the WHO (2001), focusing on biological causes for disease management singly merely leads to a 'maintenance' or 'restoration' approach to health, with no scope for improvement beyond the point of freedom from the disease.

In a patient-centred health-care set up, patients' needs are considered first and foremost at every point in the planning, implementation, and evaluation of service delivery (Thornquist 1994). For that reason, by identifying patients' health-education needs directly, a number of benefits in the management of low-back pain are likely to be realised. To address health-education needs adequately for patients with LBP, their precise needs must be known by those delivering the health education. This can only be achieved by asking the patients directly. In a study that directly sought to identify patients' information needs conducted in Norway by Glenton (2002), participants were interviewed long after completing their treatment sessions. The information that was volunteered therefore was based on memory, as participants were not experiencing the needs as they unfolded. The current study therefore aimed at determining the expressed health-education needs from the patients with LBP directly, and to use the knowledge to develop new health-education programmes for these patients.

\section{METHOD}

A qualitative research approach using in-depth interviews and one focus-group discussion was employed in order to explore the health-education needs of the individuals with LBP.

\section{Research setting}

The research was conducted at the Nairobi Hospital Rehabilitation Unit, a premier private health facility in Kenya. The facility has the resources that may be required to develop appropriate health-education programmes as expressed by the individuals unlike the under funded public-health facilities that struggle to offer services predominantly with insufficient resources.

\section{Participants' recruitment}

The study sample consisted of ten adult individuals undergoing physiotherapy treatment for LBP that was conservatively managed during the months of November 2003 and January 2004.
Members of the Rehabilitation Unit provided research participants with information about the study and enquired whether they were willing to be contacted by the researcher. In order to capture as many different aspects and needs as possible, participants were chosen strategically to include a range of background variables that could be relevant to the study.

A purposive sample was taken based on the judgement of the researcher, which embraced the most characteristic, representative, and typical attributes of the population to be studied. A short survey made up of closed or short answer questions in order to screen and to obtain expressive information about participants was used. These survey questions were based on literature. Participants then received a letter of information as well as oral information regarding the aim of the study and the method to be used. Participants were selected according to the following criteria: a representation of both genders at a certain ratio according to patient turnout at the Rehabilitation Unit, attendance of a minimum of four treatment sessions so as to capture those who had been educated on LBP, medically insured and the uninsured, employed and unemployed, and the ability to communicate in Swahili, English or both.

\section{Interviews}

Scheduled semi-structured interviews were conducted, and participants were encouraged to give their own views and needs for health education. Participants were assured that the information given would not in any way affect their treatment. During the interviews, probes were used to explore their needs in more depth and to indicate to the participant that the researcher was keenly following their responses. All the interviews were recorded on audiotapes, and participants were assured that the recordings were to be listened to by the researcher and the project supervisor. Participants would remain anonymous. Interviews were conducted until a saturation point was reached when no new data appeared to emerge.

\section{Focus-group discussion}

As a triangulation method, the focusgroup discussion was conducted with the same individuals who were involved in the face-to-face interviews at a time and place agreed upon by the participants. As a method, focus-group discussions generate a process of contribution and comparing among the participants (De Vos 2002). During the discussion, the moderator (researcher) addressed each participant by name so as to recall during transcribing which participant responded to which question. The focusgroup discussion was audio recorded and lasted one and half-hours.

\section{Ethical considerations}

All the necessary approvals were obtained from the relevant institutions. An informed consent form was signed by those willing to participate in the study. Participation in the research was voluntary and participants were allowed to withdraw their consent at any stage of the study. No names were to be revealed at any stage of the study.

\section{Data analysis}

In a phenomenology interview, ideas emerge that can better be understood under the control of a thematic analysis (Aronson 1992). The thematic analysis focused on identifying themes and patterns of expressions from the participants. The initial analysis started after the first interview and involved listening to the audiotapes several times before transcription of every interview. All transcripts and the field notes were then read and re-read several times by the researcher before assigning codes. From the transcribed conversations, patterns were listed. Category codes were included or excluded, expanded or condensed in order to respond to the themes noted within the data, guided by the aims and objectives of the study. The project supervisor independently generated categories to enhance the validity of the researcher's categorising methods. This process reduced the researcher's bias. Categories were discussed and where necessary adjusted. The degree to which the chosen categories covered all aspects of the interviews were evaluated and adjusted. A small percentage of the data was given to an independent researcher for a trustworthiness check according to the researcher's coding scheme. Any unclear codes were identified and decision rules created to clarify. Themes that emerged were pieced together to form a comprehensive picture of participants' collective expressions or experiences. While the current 
study yielded several points of great interest, those reported here are restricted to the aim and objectives of the study. One way in which credibility was established was by identifying and describing participants in the study in every respect. The data was triangulated through use of field notes, transcripts, the contribution from various participants both in the face-to-face interviews and the focus-group discussion.

\section{RESULTS AND DISCUSSION}

Most of the points of interest that emerged from the face-to-face interviews were repeated in the focus-group discussion. The two sources of data are therefore presented and discussed together. Sample quotes from the responses of the participants are provided for each theme because they express fluently the common needs among the participants. To ensure anonymity, participants' identities are replaced with codes.

The data derived from the healtheducation needs that the participants expressed fell into two main categories: that derived from the dissemination of health information and from the source of health education. Thus, deficiencies of health education were in: explanation, communication methods and skills among medical professionals, and the source of health education. Although there were variations as to the order in which the needs were expressed, these expressions displayed homogeneous patterns.

\section{Demographic profile of the participants}

The study population was by gender ratio at 4 to 6 (male to female) and employment status about 8 of them employed. Their age ranged from 27 to 52 years with an average of 40 years. With regard to education, the least educated of the participants had reached the fourth form and the most educated had a master's degree. The most surprising finding in the present study was that academic qualifications did not appear to influence the need for health education.

Lack of explanation on the cause, diagnosis, prognosis and the appropriate use of health services for LBP

The deficiencies in health education that emerged most prominently were the lack of explanation of the cause, of the diagnosis, of the prognosis, and of the appropriate use of health services for LBP. Participants in the study saw explanations of these four aspects as essential because they could lead to clarification on how to circumvent LBP, and help them to acquire self-remedying behaviours as well as in seeking health services at the right time. The findings indicate clearly that participants in the study were willing to take control of their low back pain. However, lack of clear explanation on any one or all of the four aspects of treatment was therefore likely to interfere with this resolve, and could lead to dissatisfaction with the treatment given and a possible loss of trust of the medical professional. Moreover, medical professionals could hinder the individual from achieving his or her own control of the LBP through their failure to present appropriate explanations on the four aspects of treatment. Some of the participants believed that this could lead to their undergoing inappropriate consultations.

For example, participant FI-3 stated: "They just told me it was normal to have a lower back pain ... I am not sure I really know what is causing my problem (LBP). Without a clear understanding of my LBP problem, it will be very difficult for me to manage it on my own. I will have to depend on the physiotherapists and the doctors".

Participant FI-1 was more critical describing the lack of a clear explanation on the diagnosis: "It has been a mix-up thing all through ... which at times leaves us confused. There are so many versions about my LBP that I have totally lost trust with some of the medical professionals, they are so diverse ... I lack the diagnosis of my problem".

Further expressions over the lack of explanation on the cause of LBP were well articulated by participant FI-6 who stated: "I actually was not clear on what really causes low back pain; the information I think I am lacking is the cause. What exactly is causing my lower backache? You know, once I know the cause ... the effect will not be that bad. Once I know this is what is causing my problem then I can avoid it ... that is the missing link as far as I am concerned".

Treatment outcome is a crucial factor in determining compliance levels to any treatment regime in any health condition. Prior knowledge of the prognosis encourages individuals to adhere to the prescribed treatment regime. Without the necessary information on the treat- ment outcome, little is likely to be achieved. This is widespread among individuals with chronic conditions such as LBP. As an illustration for the need on this type of information:

Participant FI-5 consequently reported: "I would like to know, is it a problem that can really go away or is it a problem that I will have to live with for the rest of my life? I would really like to be clear on that so that I can decide whether to spend on it or not'. There was more emphasis on the lack of explanation on the prognosis from a participant in the focus group FG-6: "Initially I thought this thing (LBP) was going to be over within three days ... after the commencement of physiotherapy ... I did not know that ... I was to be given something (programme) that I had to continue with at home for a long time, neither do I know up to now when this problem will go away."

The most important reason for patient consultation with any medical professional is that the patient be given an adequate explanation of the symptoms of their diagnosis, and the cause of LBP. Unfortunately, very little is known about LBP (Klaber-Moffett and Richardson 1997, Burton 1997, Waddell 1998). As a result, it is difficult to explain in pathoanatomical terms the real cause and the diagnosis, let alone the prognosis in the majority of LBP. Furthermore, LBP has been described as a multi-factorial form of illness (Waddell 1996) that requires an open approach addressing the patient's needs as expressed. However, in troublesome conditions that are difficult to explain such as LBP, Turner (1996) suggest that education about aspects of the condition such as the causes, diagnosis, and prognosis could be made effective if placed in the wider context of the individual's life. For instance, education about the cause, diagnosis, and prognosis could use the Rehabilitation Model of health education where emphasis is placed more on the patient's functioning and reduction of suffering (Sullivan, Turner \& Romano, 1991). Treatment goals can then be altered from the identification and eradication of the cause to the improvement in the patient's functioning and lessening of suffering. Health education then, according to Turner (1996), could include explanation of what was and what was not causing the patient's pain, indicating to the patient, that pain was 
mostly expected to improve over a reasonable time course.

Lack of explanation on the available health services and the appropriate use of the services were also a key concern in the present study. Lack of appropriate use of health resources was capable of hindering participants from accessing suitable health-care services. In the process, some participants may have been compelled to consult a variety of medical professionals inappropriately. The diverse medical professionals consulted were also likely to offer incoordinated treatment, advice and could, as well, expose individuals to conflicting health education or could lead them to a delay in accessing appropriate health service (Fritz and George 2002, Lee et al 2004) leading to high financial consequences to the individuals and loss of confidence with the health services. A case in point was the expression by participant FI-3 who reported: "I have gone to an orthopaedic doctor ... Chinese herbalist and a chiropractor. Actually I have a problem... I don't know whether to see a gynaecologist ... or another orthopaedic doctor... we don't know... people don't know exactly whom to see ... We, people don't really know ... yet nobody appears to explain to us the most appropriate medical personnel to consult ".

Further expressions regarding explanation over the appropriate use of health services came from participant FI-7 who stated: "many people lack all the information ... because they don't know what to do once someone is faced with that kind of a situation (LBP) ... they will therefore go to any medical practitioner. We need to be informed where to go for back treatment".

This point was highlighted by a participant in the focus-group discussion FG-7: "I had not heard about physiotherapy before, people don't know what physiotherapy is ... I think just like me, there are many people out there who do not know what physiotherapy is and who may benefit from the service".

An expression that makes clear the need for explanation on the appropriate use of health services was captured from participant FI-7 who stated: "many people lack all the information ... because they don't know what to do once someone is faced with that kind of a situation (LBP) they move from one medic to another who rarely mentions physio- therapy unless the individual has prior knowledge of physiotherapy services".

According to personal experience by the authors, in many developing countries, a number of health programmes including physiotherapy were until recently a preserve of the urban rich and at times, were viewed as a luxurious form of health service. This has had a negative effect on the accessibility to these programmes by deserving individuals.

Furthermore, despite the decentralisation of the health services in Kenya for more than four decades now with emphasis to rural areas, physiotherapists have not managed to popularise physiotherapy programmes to the general population. Most patients are therefore have remained unfamiliar with this form of health programme and are for that reason unable to consult appropriately when faced with a LBP problem. The fact that health-service advertisement is not allowed could be the reason for this finding among the less-educated rural population. Additionally, physiotherapists may be unfamiliar with modern marketing strategies allowed in the health service that may have enabled the general population to be knowledgeable on matters of health programmes within their respective areas.

Nonetheless, according to Pitt-Brooke et al (1998), one type of health education aims at providing patients with information about the health resources available and the sensible use of those services to their advantage. Once familiar with the health services, the medical professionals ought to encourage the individuals to make correct and timely use of the health resources when necessary so as to assist in minimising delay in seeking for an appropriate health service.

\section{Lack of appropriate communication methods and skills}

Most of the participants in this study reported to have received verbal health information regarding their low-back pain. They complained of a paucity of written health information regarding LBP in several health-care facilities. In addition, due to the verbal method of dissemination of information, participants felt there was a lack of consistency among physiotherapists and that such a method of dissemination often led to forgetfulness on the participants' part. Moreover, the verbal method lacks stan- dardisation among physiotherapists and as a result, is prone to misuse. It is therefore unsuitable for the participants. The verbal method of communication renders the information disseminated informal and thus is likely to be a source of confusion, and conflict between patients and physiotherapists.

For example, participant FI-6 asserted: "I think it should be written ... because these verbal things (verbal method of education) especially at my age (at 52) ... now I tend to forget a lot of things. Put down in writing in a pamphlet form and make it graphic ... you see verbal communication varies on what a person remembers or what a person knows. So if you have it written in a diagrammatic form ... it's the right way I suppose".

Another participant FI-2 confirmed this: "it (health education) was a word of mouth, (pauses) ... he told me how to do it (exercise) ... just talking when I go for my clinics he (the gynaecologist) continues to ask me how I am managing. I was actually given nothing else".

The material presented verbally also raised questions about its relevance since such information materials are, on most occasions, based on personal experiences, opinions, and beliefs. Health education needs to be effective both in terms of the methods used and the content presented. This can only be achieved by drawing on research knowledge and critical evidence-based actions. The information should then be standardised both in content and the mode of presentation. Furthermore, there is evidence to suggest that appropriate communication methods such as leaflets have more benefits for patients than verbal communication. (Symonds et al 1996). Such an approach would be more appropriate for patients and in addition, it would be a standard method of disseminating information by different physiotherapists. This seems desirable since many health practices today include health-education programmes based on experience, traditional models, and untested theories (Kitson et al 1996, French 1999).

A further crucial factor that has influenced health education negatively is the lack of appropriate communication skills among the medical professionals. Participants in the current study described the lack of appropriate commu- 
nication skills among medical professionals as a hindrance to their health education. They reported that they were at times unable to understand what the medical professionals meant by certain terms. The language used was described as technical which participants considered unsuitable for communicating with them. On many occasions, participants did not understand the advice given by medical professionals because of the use of technical terms.

For instance, participant FI- 4 said: "Some of the problems I encountered were some of those words that were used ... were a bit hard (technical) for me to comprehend. And that could not make it so easy for me to understand fully some of the terminologies used. In some cases they were using very technical words".

Participant FI-2 had a similar problem: "When he said disc disease, I asked him what he meant, he told me ... 'the disc has a disease rather' Well to me it was the same as what he had said ... I never understood what was meant to this day".

Further emphasis over the same concern came from a participant in the focus-group discussion FG-5 who said: "The physiotherapist told me I am suffering from a condition called ... was it ... lumbago ... or something like that ... I am not very sure of the word but that was what the physiotherapist said"

In other studies, it has also been found that, verbal information from doctors, nurses, and physiotherapists has often been too technical for a large number of non-medical people, including patients, to understand (Davis et al 1996). Consequently, the use of technical terms by medical professionals suggests the inability to communicate in simple, easy language to their patients, which is a key component of an effective healthcare service (Elstein \& Schwarz 2002). An effective therapeutic alliance is based on good communication skills. In view of the findings, there is a need for medical professionals to improve on communication skills so as to communicate successfully with their patients for a positive treatment outcome.

That poor communication skill among medical professionals should be an occasional but constant cause of dissatisfaction among patients is an area of much concern. However, despite the evidence and the fact that physiothera- pists are aware of the difficulties involved in explaining medical terminologies to their patients in easy layman's terms (Sluijs et al 1993), it appears that this matter has not been addressed effectively. The need for effective communication is further advanced by the emerging models of health-care delivery, which have a strong fundamental reliance on successful interpersonal communication skills, not only between patients and health practitioners, but also among those practising within the health sector (Nordholm et al 1995). As health professionals, it has become important to improve communication skills as we need to determine how patients view their condition and also what there expected outcome of management would be.

\section{Lack of a Reliable Source of Health Education}

The sources of the health education that participants had received were amazingly diverse. The number of many different contributors of health information to the participants further suggests the possibility of conflicting health information disseminated to the participants. It emerged for example, that there was a lack of coordination in the way the information was presented, in the standardisation of the contents of the health information given, and in the dissemination methods from the different sources that offered the education. The most common sources from whom participants sought advice about their health and from whom they received health information included doctors, physiotherapists, nurses, friends, parents, other patients, herbalists, the Internet and books. As the information was mostly verbal it was as diverse as its sources. Moreover, some participants reported to have been exposed to conflicting health-education information from some of the sources.

For example one participant had the following contributions to make: "At first I was told by my doctor that maybe I am not fit ... so I decided to join a club or do some aerobics to be fit but (laughs) I think after ... may be two ... three sessions the back (pain) was too much. Then I was told by the physiotherapist " no you should never do exercises when you are in pain". You see, those kind of conflicting ideas. So you ... pauses ... you are left feeling that you don't know anything about how to manage your ... back" (FI-3).

Participant FI-4 reported: "I read in the Internet because when this low back pain became a problem I realised I wanted to have some more information ... about back care but even that information was not the same. It varied".

Participant FG-3 elaborated further by reporting: "One problem was that I had to visit different doctors and I would get ... you know ... conflicting kind of ... ideas (health education), some would think I had arthritis then when they take me for tests there's no arthritis, others would think that, pauses ... there were fibroids while other books said I should avoid taking medicines"

Another participant volunteered another source: "My friends told me that the other possible cause of my backache could have been too much sex" (FG-5).

The findings indicate that various sources consulted by patients will offer diverse health information based on the speciality and to some extent, personal experience of the source.

Additionally, the immense autonomy enjoyed by medical practitioners and the degree of flexibility allowed in the medical profession coupled with the lack of policies and standards in the majority of health-care institutions has contributed to the lack of standardised health information to the participants. However, when health information is presented verbally, it not only raises questions about its relevance but also lack standards among physiotherapists and other medical professionals. This is because such information is in most occasions based on personal experiences and beliefs. Furthermore, it is still said that many health practices today including health education programmes are based on experience, traditional models and untested theories (Kitson, Ahmed, Harvey, Seers \& Thompson, 1996; French, 1999). It is therefore necessary to have a central, reliable source of health information for individuals with LBP. The information should be credible based on current research findings and should be presented in a written form.

\section{CONCLUSION}

In confirming the effects of the existing medical model of health education, the findings suggest there is a need to 
address additional aspects that are not clinically related when planning healtheducation programmes for individuals with LBP. The findings moreover identify areas that require to be targeted in interventions that aim at influencing LBP positively among the individuals. Based on what participants in the current study have expressed, a paradigm shift was required so as to develop a new health education programme for the individuals. A more encompassing, all-inclusive model of health education that would cover on the aspects that have been identified was essential. A Rehabilitative Model of health education was probably more pertinent to the individuals. It was essential that new determined efforts and approaches be directly put in place targeting areas that participants have expressed as requiring attention.

\section{ACKNOWLEDGEMENT}

The Nairobi Hospital-Kenya financed this project. The authors therefore take the earliest opportunity to express their gratitude to the board of management of the Nairobi hospital for the financial assistance.

\section{REFERENCES}

Altmaier E, Russell D, Feng Kao C, Lehmann T, Weinstein J 1993 Role of self-efficacy in rehabilitation outcome among chronic back pain patients. Journal of Counselling Psychology 40: 335-339

Anderson K, Dowds B, Pelletz R, Edwards T, Peeters-As-dourian C 1995 Development and initial validation of a scale to measure self-efficacy beliefs in patients with chronic pain. Pain 63: 77-84

Aronson J 1992 The interface of family therapy and a juvenile arbitration and mediation program. Unpublished doctoral dissertation, Nova South Eastern University: Fort Lauderdale.

Axelsson A, Nilsson S, Coles R 1995 Tinnitus information: a study by questionnaire. Audiology 34: $301-310$

Bandura A 1997 Self-Efficacy: The Exercise of Control. Freeman, New York

Bardin L 2002 Physiotherapy and low back pain: part I Outcomes Research in the quest for evidence. South African Journal of Physiotherapy 58: 3-7

Bigos S, Bowyer O, Brean G 1994 Acute Low Back Problems in Adults. Clinical Practice Guideline number 14: Rockville, Md: Agency for Health Care Policy and Research publication number 95-0642, US Department of Health and Human Services, pp 1-160, AHCPR publication, New York

Burton A, Tillotson K, Main C 1995 Psychosocial predictors of work loss in acute and subchronic low back trouble. Spine 20: 722-728

Burton A 1997 Spine Update- Back Injury and Work loss: Biomechanical and Psychosocial Influences. Spine 22: 2575-2580
Cohen J, Frank J, Bombardier C, Guillemin F 1994 Group education interventions for people with low back pain. An overview of literature. Spine 19: 1214-1222

Coulter A, Entwistle V, Gilbert D 1998 Informing patients. An Assessment of quality of Patient Information Materials. King's Fund, London

De Vos A 2002. Research at Grass roots for the Social Sciences and Human Service Professionals, 2nd Edition. Van Schaik, Pretoria

Davis T, Bocchini J, Fredrickson D 1996 Parent comprehension of polio vaccine information pamphlets. Paediatrics 97: 804-810

Deyo R, Phillips W.1996 Low back pain: a primary care challenge. Spine 21: 2826-2832

Elstein A, Schwarz A 2002 A clinical solving and diagnostic decision-making; selective review of the cognitive literature. British Medical Journal 324: 729- 732

French, P. 1999. The development of evidencebased nursing. Journal of Advanced Nursing. 29: 72-78.

Fritz J, George S 2002 Identifying psychosocial variables in patients with acute work-related low back pain: The importance of fear- avoidance beliefs. Physical Therapy 82: 973-983

Glenton C 2002 Developing patient-centred information for back pain sufferers. Health Expectations 5: 1-11.

Gronblom-Lundstrom L 1992 Health as a measure of rehabilitation: outcome for patients with low back pain. Physiotherapy Theory and Practice $8:$ 67-77

Harland N, Lavallee D 2003 Biopsychosocial management of chronic low back pain patients with psychological assessment and management tools. Physiotherapy 89: 305-312

Hunt A, Adamson B, Harris I 1998 Physiotherapists' perceptions of the gap between education and practice. Physiotherapy Theory and Practice 14: 125-138 Jensen M, Turner J, Romano J 1994a Correlates of improvement in multidisciplinary treatment of chronic pain. Journal of Clinical Psychology 62: 72-179 Jensen M, Turner J, Romano J, Lawler B 1994b Relationship of pain-specific beliefs to chronic pain adjustment. Pain 57:301-309

Kitson A, Ahmed L, Harvey B, Seers K, Thompson D 1996 From research to practice: one organizational model for promoting research-based practice. Journal of Advanced Nursing 23: 430-440

Klaber-Moffett J, Richardson P 1997 The influence of the physiotherapist-patient relationship on pain and disability. Physiotherapy Theory and Practice 13: $90-96$

Klenerman L, Slade P, Stanley I 1995 The prediction of chronicity in patients with an acute attack of low back in a general practice setting. Spine 20: 478-484

Koes B, Tulder M, Van der Windt A, Bouter L 1994 The efficacy of back schools: A review of randomised clinical trials. Journal of Clinical Epidemiology 47 : 851-862

Lee S, Arozullahb A, Choc I 2004 Health literacy, social support, and health: a research agenda. Social Science and Medicine 58: 1309-1321

Leininger M 1985 Ethnography and ethno-nursing: Models and modes of qualitative data analysis. In M.M. Leininger (Ed.), Qualitative Research Methods in Nursing pp. 33-72. Grune \& Stratton, Orlando

Li L, Bombardier C 2001 Physical Therapy of low back pain: An exploratory survey of therapist approaches. Physical Therapy 81: 1018-1027
Linton S, van Tulder M 2001 Preventive interventions for back and neck pain problems: what is the evidence. Spine 26: 778-787

Maddux J Ed. 1995 Self-efficacy, adaptation, and adjustment: Theory, research, and application. Plenum, New York

Moseley L 2002 Combined physiotherapy and education is efficacious for chronic low back pain. Australian Journal of Physiotherapy 48: 297-302

Nordholm L, Adamson B, Heard R 1995 Australian physiotherapists' and occupational therapists' views on professional practice. Journal of Allied Health 24: 267-282

Pinnington M 2001 'Why are we finding it so hard to change our approach to low back pain?' Physiotherapy 87: 58-59

Pitt-Brooke J, Reid H, Lockwood R, Kerr K 1998 Rehabilitation of Movement: The Basis of Clinical Practice. Health Education \& Communication. WB Saunders, London

Polit D, Hungler P 1995 Nursing Research: Principles and Methods (6th ed), Lippincott Company, Philadelphia

Richardson B, Eastlake A 1994 Physiotherapy in Occupational Health, Management, Prevention and Promotion in the work place. Health Promotion and Education. Butterworth-Heinemann, Oxford

Roland M, Morris R 1983 A study of the natural history of low back pain: Part II. Development of guidelines for trials of treatment in primary care. Spine 8: 145-150

Royal College of General Practitioners 1996 Clinical Guidelines for the management of Acute Low Back Pain. London

Skevington S 1995 Psychology of Pain. John Wiley \& Sons, Winchester

Sluijs E 1991 A checklist to assess patient education in physical therapy: Development and reliability. Physical Therapy 71: 561-569

Sluijs E, Van der Zee J, Kok G 1993 Differences between physical therapists in attention paid to patient education. Physiotherapy Theory and Practice 9:103-117

Sullivan, M D, Turner, J A \& Romano J (1991). Chronic pain in primary care. Identification and management of psychosocial factors. Journal of Family Practice. 32: 193-198.

Symonds T, Burton A, Tillotson K, Main C 1996 Absence resulting from low back trouble can be reduced by psychosocial intervention at the work place. Spine 20: 2738-2745

Thornquist E 1994 Profession and life: separate worlds. Social Science Medicine 39: 701-713

Turner J 1996 Education and behavioural interventions for low back pain in primary care. Spine 21: 2851-2859

Von Korff M, Moore J, Loring K, Cherkin D, Saunders K, Gonzales V, Laurent D, Rutter C, Comite F 1998 A randomised trial of a lay personal-led self-management group intervention for back pain patients in primary care. Spine 23: 2608-2615

Waddell G 1996 Keynote address for primary care forum. 'Low back pain: A twentieth-century health care enigma'. Spine 24: 2820-2825

Waddell G 1998 The back pain revolution. Psychosocial Factors of Low Back Pain. Churchill Livingstone, London

World Health Organisation 2001 World health assembly for international use. World Health Assembly 54: 21 